\title{
Stimulated Brillouin scattering suppression with a chirped laser seed: comparison of dynamical model to experimental data
}

Eliot Petersen, Zhi Yang, Jeffrey O. White, George Rakuljic, Naresh Satyan, et al.

Eliot Petersen, Zhi Yang, Jeffrey O. White, George Rakuljic, Naresh Satyan, Arseny Vasilyev, Amnon Yariv, "Stimulated Brillouin scattering suppression with a chirped laser seed: comparison of dynamical model to experimental data," Proc. SPIE 8961, Fiber Lasers XI: Technology, Systems, and Applications, 89613H (17 March 2014); doi: 10.1117/12.2037750 


\title{
Stimulated Brillouin scattering suppression with a chirped laser seed: Comparison of Dynamical Model to Experimental Data
}

\author{
Eliot Petersen $*^{\mathrm{a}}$, Zhi Yang ${ }^{\mathrm{a}}$, Jeffrey O. White ${ }^{\mathrm{a}}$, \\ George Rakuljic ${ }^{\mathrm{b}}$, Naresh Satyan ${ }^{\mathrm{b}}$, Arseny Vasilyev ${ }^{\mathrm{c}}$, Amnon Yariv ${ }^{\mathrm{c}}$ \\ a Army Research Laboratory, 2800 Powder Mill Rd., Adelphi, MD, USA 90783; \\ ${ }^{\mathrm{b}}$ Telaris, Suite 238, 2118 Wilshire Blvd, Santa Monica, CA USA 90403 \\ ${ }^{\mathrm{c}}$ California Institute of Technology, Pasadena, CA USA 91125
}

\begin{abstract}
When scaling CW single-mode fiber amplifiers to high power, the first nonlinear limitation that appears for narrowlinewidth seed lasers is stimulated Brillouin scattering (SBS). We present a dynamical simulation of Brillouin scattering in a $\mathrm{Yb}$-doped fiber amplifier that numerically solves the differential equations in $z$ and $t$ describing the laser, Stokes and pump waves, the inversion, and the density fluctuations that seed the scattering process. We compare the model to experimental data, and show that a linearly chirped seed laser is an efficient form of SBS suppression; especially for long delivery fibers. The frequency chirp decreases the interaction length by chirping through the Brillouin resonance in a time that is short compared to the fiber transit time. The seed has a highly linear chirp of $10^{14}-10^{16} \mathrm{~Hz} / \mathrm{s} \mathrm{at} 1064 \mathrm{~nm}$ which preserves a well-defined phase relationship in time. This method of SBS suppression retains a long effective coherence length for purposes of coherent combining, while at high chirps appears to the SBS as a large linewidth, increasing the threshold. An increase in fiber length increases the laser bandwidth as seen by the SBS, leading to a fiberlength-independent SBS threshold.
\end{abstract}

Keywords: stimulated Brillouin Scattering, fiber amplifiers, frequency chirped laser

\section{INTRODUCTION}

There is a need for high-power single-mode fiber amplifiers that can maintain narrow linewidth operation to enable relatively straightforward coherent combining of multiple amplifiers. An electronically-controlled phased array would enable pre-compensation for atmospheric turbulence; whereas in spectral beam combining and non-coherent combination this would require a deformable mirror. The usual method of obtaining high output from fiber amplifiers is to broaden the linewidth to avoid fiber nonlinearities e.g., Brillouin scattering. Instead of broadening the seed laser linewidth we apply a deterministic frequency chirp to the seed laser before amplification. This chirped seed laser presents itself as broadband to the backwards propagating Stokes wave generated through stimulated Brillouin scattering (SBS). When using this source, different amplifier path lengths translate into different frequencies at the output of each. Thus, different amplifier lengths can be compensated by preceding each amplifier with a variable frequency shifter, and controlling it with a feedback loop. An important step in realizing such a system is to calculate the SBS threshold for a single amplifier seeded with a chirped laser. The next section describes a numerical model of an amplifier that can be seeded with an arbitrary waveform, allowing the performance of chirped and conventional seeds to be compared.

\section{NUMERICAL MODEL}

Previously, many models describing Brillouin scattering have assumed a weak Stokes wave seed incident at the output end of the fiber instead of using a more physical stochastic seeding distributed along the length of the fiber [1]. This can be a valid simplification if there is no variation in the Brillouin frequency shift along the length of the fiber, but breaks

Fiber Lasers XI: Technology, Systems, and Applications, edited by Siddharth Ramachandran,

Proc. of SPIE Vol. 8961, 89613H · C 2014 SPIE · CCC code: 0277-786X/14/\$18 doi: $10.1117 / 12.2037750$ 
down under longitudinal thermal or stress gradients, or for a linear chirp of the seed laser. We include the distributed nature of the seeding process by adding a white noise source $(f)$ to eq. $4[2,3]$.

A valid assumption is neglecting the propagation of the phonons within the glass fiber. This is valid as the glass matrix limits their lifetime to $\sim 20 \mathrm{~ns}$ and thus their propagation distance to a tenth of a $\mathrm{mm}$. This makes the density variation a function of only the local electric fields.

Our simulation of SBS is based on solving the following coupled differential equations: [4]

$$
\begin{gathered}
\frac{\partial E_{L}}{\partial z}+\frac{n}{c} \frac{\partial E_{L}}{\partial t}=\frac{-\alpha_{L}(z)}{2} E_{L}+i \kappa E_{S} \rho, \\
-\frac{\partial E_{S}}{\partial z}+\frac{n}{c} \frac{\partial E_{S}}{\partial t}=\frac{-\alpha_{S}(z)}{2} E_{S}+i \kappa E_{L} \rho^{*}, \\
\frac{\partial E_{P}}{\partial z}+\frac{n}{c} \frac{\partial E_{P}}{\partial t}=\frac{-\eta \alpha_{P}}{2} E_{P}, \\
\frac{\partial \rho}{\partial t}+\pi \Delta v_{B} \rho=i \Lambda E_{L} E_{S}^{*}+f, \text { and } \\
\frac{\partial N_{2}}{\partial t}=\frac{c n \varepsilon_{0}}{2 h}\left(\frac{\left|E_{P}\right|^{2} \alpha_{P}(z)}{v_{P} A_{\text {Clad }}}+\frac{\left|E_{L}\right|^{2} \alpha_{L}(z)}{v_{L} A_{\text {Core }}}+\frac{\left|E_{S}\right|^{2} \alpha_{S}(z)}{v_{S} A_{\text {Core }}}\right)-N_{2} W_{21}
\end{gathered} .
$$

$E_{L}, E_{S}$, and $E_{P}$ are the slowly-varying envelopes (amplitudes) of the laser, Stokes and pump fields, $\rho$ is the amplitude of the density fluctuations in the fiber core, and $N_{2}$ is the excited state ion population density. The pump and signal transverse spatial overlap is given by $\eta$, and the spontaneous emission rate is $W_{21}$. The laser, pump, and Stokes frequencies are $v_{\mathrm{L}, \mathrm{P}, \mathrm{s}}$; the core and cladding cross-sectional areas are $\mathrm{A}_{\text {Clad, core. }}$ The function $f$ describes the thermal phonons that are responsible for spontaneous Brillouin scattering. $\kappa$ and $\Lambda$ are the optic and acoustic coupling parameters. The laser, Stokes, and pump gain/absorption are defined as $\alpha_{L, S, P}$.

The above equations were solved numerically using the method of characteristics. The boundary condition on the laser field is $E_{L}(0, t)=E_{0} \exp \left[i \pi \beta t^{2}\right]$ where $\beta$ is the chirp [5]. The initial condition has no optical fields within the fiber. At $t=0$ the pump enters the fiber at $z=0$ and/or $z=L$ and the laser enters at $z=0$. There is no input Stokes wave, i.e. $E_{S}(L, t)=0$. Pseudo-equilibrium is typically reached after $\sim 10-100$ transit times. The step size was determined empirically by decreasing it until the values of the average Stokes power converged.

Fig. 1 is a snapshot in time of the Stokes power vs. position in a passive LMA fiber and shows fluctuations along the length of the fiber. In this simulation, $\Delta v_{B}=50 \mathrm{MHz}, g_{0}=2 \mathrm{~cm} / \mathrm{GW}$, and the laser input power is $50 \mathrm{~W}$. For zero chirp, the process is far above threshold, thus a large fraction of the input laser power is reflected. A chirp of $10^{16} \mathrm{~Hz} / \mathrm{s}$ brings the Brillouin scattering back to the spontaneous level. One can see that the length scale over which SBS builds up is in agreement with the effective length defined in [5] as $L_{\text {eff }} \cong \pi \Delta v_{B} c / 4 n \beta$. 


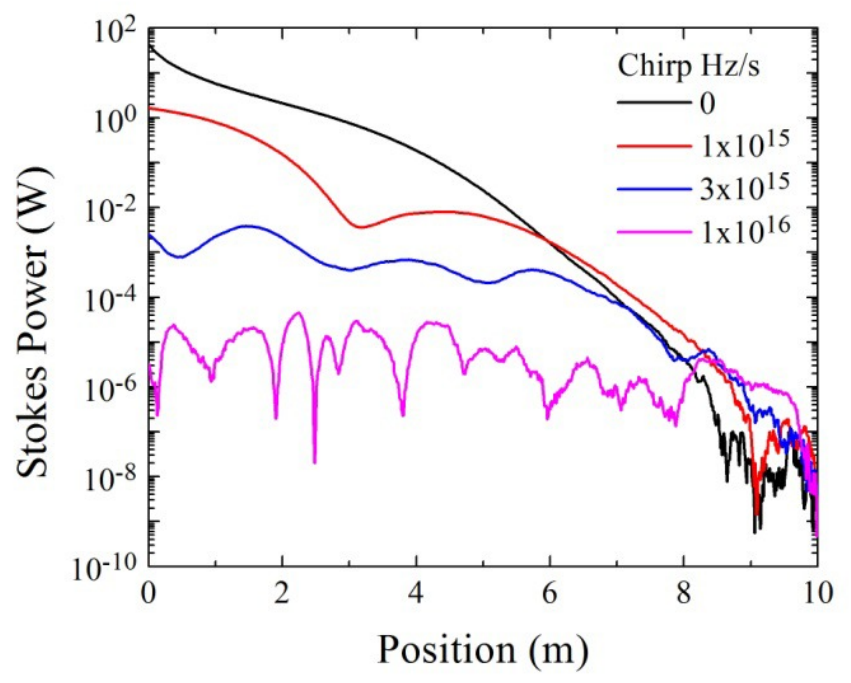

Fig. 1. Stokes power along the length of a passive fiber, recorded at one time step, for various chirps. The laser wave propagates from left to right. The Stokes wave propagates from right to left, and builds up from a distributed, stochastic source.

\section{EXPERIMENTAL VALIDATION}

The seed for our experiments is a $1064 \mathrm{~nm}$ diode laser chirping at $1 \cdot 10^{14}-4 \cdot 10^{16} \mathrm{~Hz} / \mathrm{s}$ [6]. Data is first taken with a $5 \mathrm{~m}$ $\mathrm{Yb}$ fiber amplifier with a $41.5 \mathrm{~m}$ delivery fiber spliced on. Both active and passive fiber are Nufern polarizationmaintaining $25 / 400$ fiber, with an NA $=0.065$. The backscattered power and output power are measured with a tap and photodiodes (Fig. 2.)

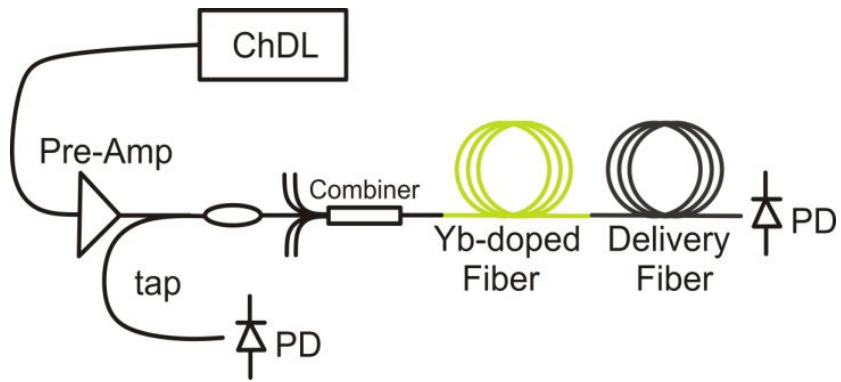

Fig. 2. Experimental setup for Brillouin scattering measurement, including chirped diode laser (ChDL), $0.05 \%$ backwards tap, pump and signal combiners, photodiodes for monitoring forward and backwards power.

Figure 3 shows the backscattered power from the final stage vs. output power, for various chirps. The dots are experimental data; the lines are results from the simulation. The measured backscattered power includes Rayleigh and Brillouin scattering, and Fresnel reflections from splices or other components. Below the SBS threshold, Rayleigh scattering dominates. At zero chirp, we can measure the Rayleigh and Brillouin components separately, using an optical spectrum analyzer (OSA). 


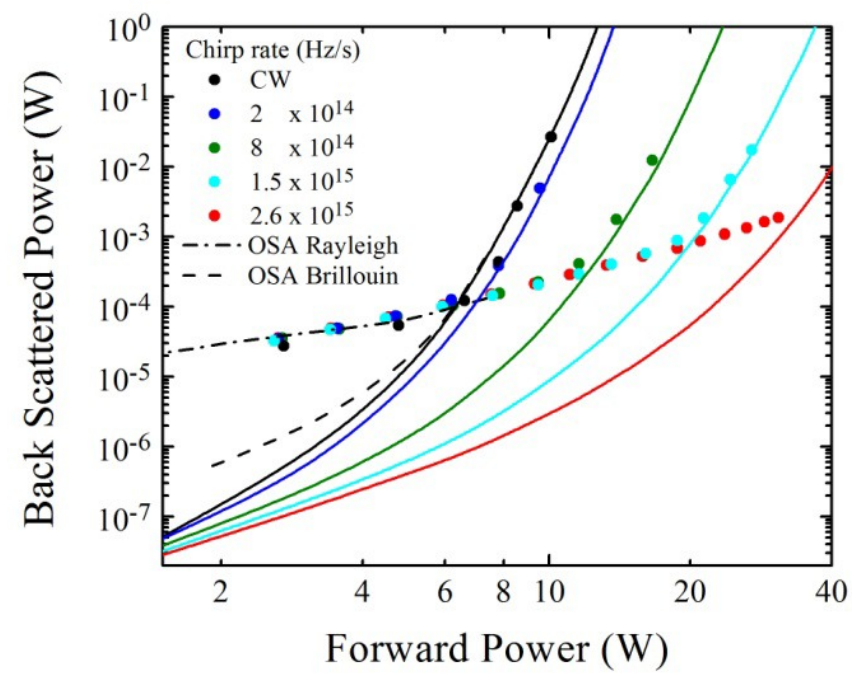

Fig. 3. Backscattered power vs forward power for various chirps, for a $5 \mathrm{~m}$ active fiber and a $41.5 \mathrm{~m}$ delivery fiber. The points are experimental data; the solid lines are results of the simulation. The dashed (Brillouin) and dash-dot (Rayleigh) lines are measured at zero chirp with an optical spectrum analyzer.

Table I contains the parameters used in the simulation. They are literature values except for the Brillouin bandwidth $\left(\Delta v_{\mathrm{B}}=140 \mathrm{MHz}\right)$ and gain $\left(g_{\mathrm{B}}=1.89 \times 10^{-11} \mathrm{~m} / \mathrm{W}\right)$ which were varied to fit the data.

Table I

Simulation Parameters

\begin{tabular}{||ll||ll||}
\hline$\gamma$ & 0.902 & $\rho_{0}$ & $2210 \mathrm{~kg} / \mathrm{m}^{3}$ \\
$v$ & $5960 \mathrm{~m} / \mathrm{s}$ & $\mathrm{A}_{\text {Core }}$ & $\pi(13.5 \mu \mathrm{m})^{2}$ \\
$\mathrm{n}$ & 1.45 & $\mathrm{~A}_{\text {clad }}$ & $\pi(200 \mu \mathrm{m})^{2}$ \\
$\mathrm{~T}$ & $300 \mathrm{~K}$ & $\lambda_{\mathrm{L}}$ & $1065 \mathrm{~nm}$ \\
$\mathrm{~N}_{\mathrm{Yb}}$ & $8.0 \times 10^{25} \mathrm{~m}^{-3}$ & $\lambda_{\mathrm{P}}$ & $976 \mathrm{~nm}$ \\
$\Delta v_{\mathrm{B}}$ & $140 \mathrm{MHz}$ & $\mathrm{g}_{\mathrm{B}}$ & $1.89 \times 10^{-11} \mathrm{~m} / \mathrm{W}$ \\
$\Delta v_{\mathrm{L}}$ & $50 \mathrm{MHz}$ & & \\
\hline
\end{tabular}

A second experiment was performed with a non-PM amplifier having a $10 \mathrm{~m}$ active fiber and a $2 \mathrm{~m}$ delivery fiber. The core diameter, cladding diameter, and NA are the same as the previous experiment. As before, the backscattered power is dominated by Rayleigh scattering below SBS threshold. The backscattered power rises approximately linearly in this region, and then rises steeply above SBS threshold (Fig. 4). The dashed line indicates a backward power equal to $0.01 \%$ of the output power. Using that as a definition of SBS threshold, a chirp of $4 \cdot 10^{16} \mathrm{~Hz} / \mathrm{s}$ increases the threshold $\sim 10 \times$ compared to the unchirped case. At the maximum chirp, the output power is limited by the available pump power. The solid lines in Fig. 4 show the results of a simulation, using the same parameters as in Table I. Above threshold, the model agrees well with the data. At the highest chirp, the threshold is becoming proportional to chirp. 


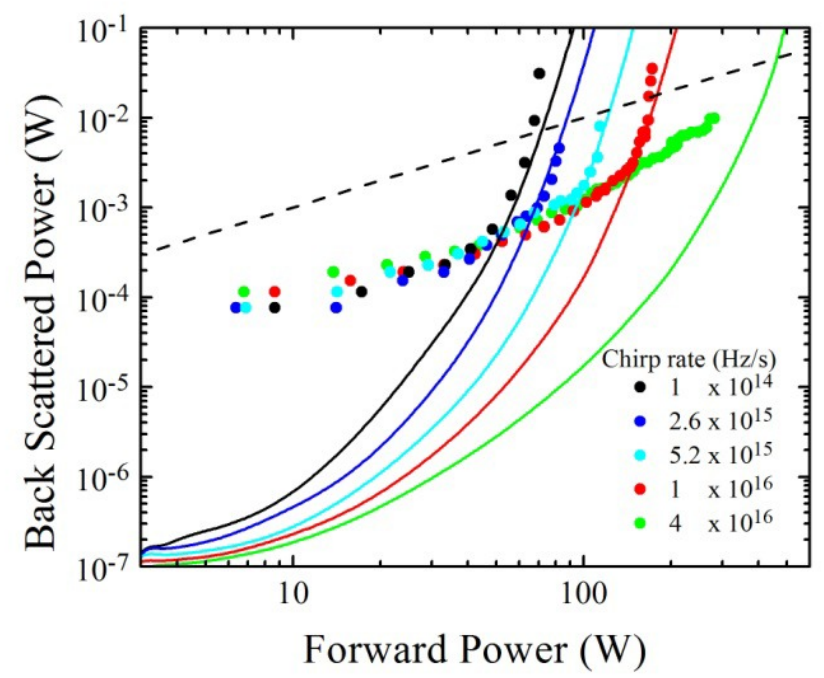

Fig. 4. Experimental points of the total backscattered light and modeled lines of the Brillouin scattering component for different chirp rates. The data and simulation were done for a $10 \mathrm{~m}$ active fiber and a $2 \mathrm{~m}$ delivery fiber.

\section{SBS THRESHOLD SCALING WITH CHIRP AND LENGTH}

As the chirp increases it acts to decrease the interaction length of the laser and Stokes wave. Once this effective interaction length is appreciably shorter than the actual fiber length the SBS threshold increases linearly with chirp (Fig. 5). This simulation was run for a non-PM $25 / 400$ fiber amplifier having a $10 \mathrm{~m}$ active fiber length and a variable delivery fiber length. The parameters in Table I were used and the threshold level was defined as $0.01 \%$ of the amplifier output power.

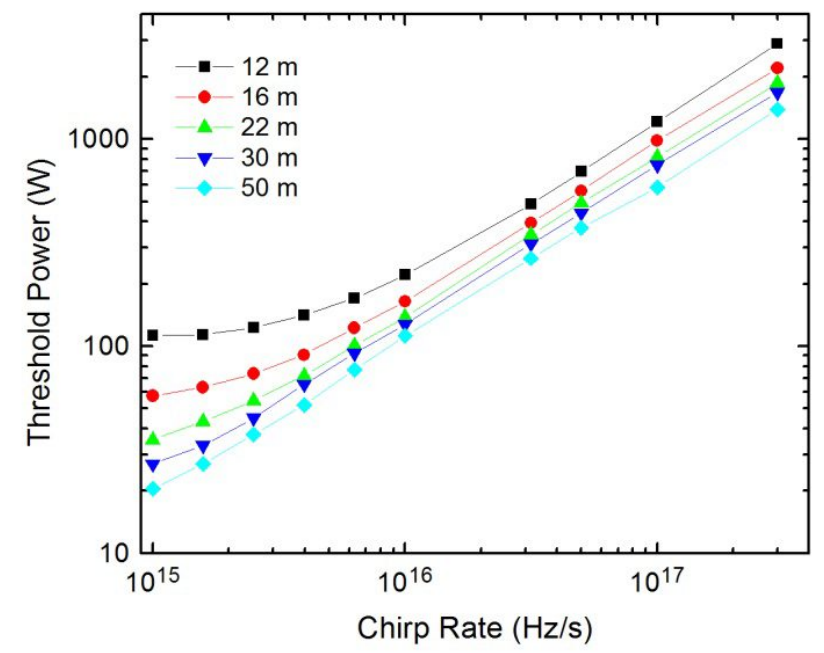

Fig. 5. SBS threshold vs. chirp for different total fiber lengths (active + delivery) in a Yb amplifier. The simulation is for a $10 \mathrm{~m}$ active fiber and a variable length delivery fiber using the parameters in Table I.

This closely follows the linear increase in threshold with chirp that was described in reference [5]. This is because the effective laser bandwidth, i.e. the bandwidth seen by the counter-propagating Stokes wave, is proportional to fiber 
length. There is, however, a small decrease in threshold with fiber length even for high chirps. This is due to the additional spontaneous scattering from a longer fiber, and is unavoidable.

The simulation was also used to compare SBS suppression between a random phase modulation and the linear chirp. To simulate a spectrally broadened seed source, we added at each time step a small random phase shift to the laser electric field at $\mathrm{z}=0$. The spectrum was fit to a Lorentzian to obtain the FWHM. The operating curve for a $26 \mathrm{GHz}$ random phase modulation is shown along with 4 values of the linear chirp in Fig. 6. The threshold for a linear chirp decreases substantially slower than $1 / \mathrm{L}$, whereas the threshold for random modulation decreases faster than $1 / \mathrm{L}$. Our simulation of random phase modulation agrees well with the $1.4 \mathrm{~kW}$ threshold obtained with a $25 \mathrm{GHz}$ (FWHM) seed bandwidth [7].

The maximum phase modulation achievable with a single electro-optic modulator is $\sim 25 \mathrm{GHz}$, limiting this method to an SBS-free output on the order of a kW. In addition, a modulation bandwidth comparable to the Stokes shift (16 GHz) raises the possibility of self-seeding the Stokes wave because of backward Rayleigh scattering. This possibility can be completely avoided in our approach by using a negative linear chirp.

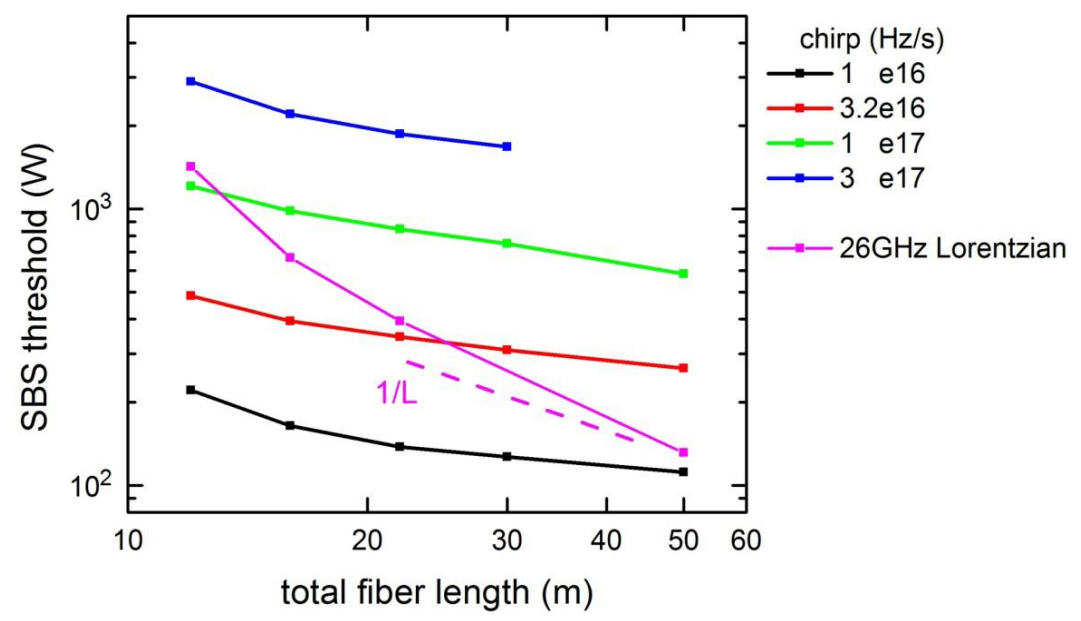

Fig. 6. SBS threshold vs. total fiber length (active + delivery) for different chirps and a $26 \mathrm{GHz}$ random phase modulation.

\section{SBS POWER VARIATION WITH SEED LEVEL}

The numerical calculation of the Stokes wave amplitude at any point in the fiber is useful as it allows us to visualize the SBS dynamics and adjust parameters in order to minimize the Stokes gain. As the laser seed power is reduced the inversion near $z=0$ increases, leading to more gain for the backwards Stokes wave. On the other hand, as the seed power is increased the actual laser power along the length of the amplifier increases leading to higher Brillouin gain as well. This means there is an optimum seed power that minimizes the Stokes power at $z=0$. Figure 7 shows the Laser and Stokes power vs. longitudinal position along the fiber for various laser seed powers. The model was averaged over 5 transit times for each seed power to reduce the noise in the Stokes signal. 


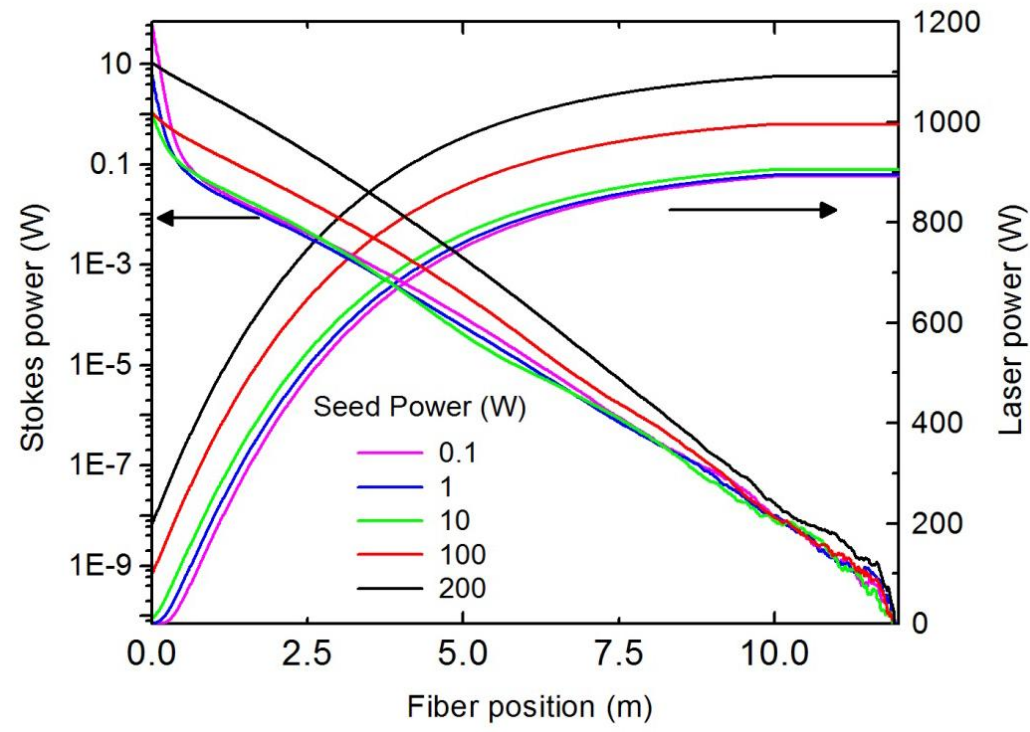

Fig. 7. Stokes and Laser power vs. longitudinal position along fiber for a chirp of $5 \cdot 10^{16} \mathrm{~Hz} / \mathrm{s}$ and a $\mathrm{kW}$ of co-propagating pump power, averaged over five transit times.

We ran the model for a number of seed powers to determine the seed power that minimizes the Stokes power at $z=0$. Figure 8 shows a minimum in the SBS power at a laser seed power of $30 \mathrm{~W}$ for a $10 \mathrm{~m} \mathrm{Yb}$-doped active fiber, $2 \mathrm{~m}$ delivery fiber, both consisting of 25/400 non-PM fiber.

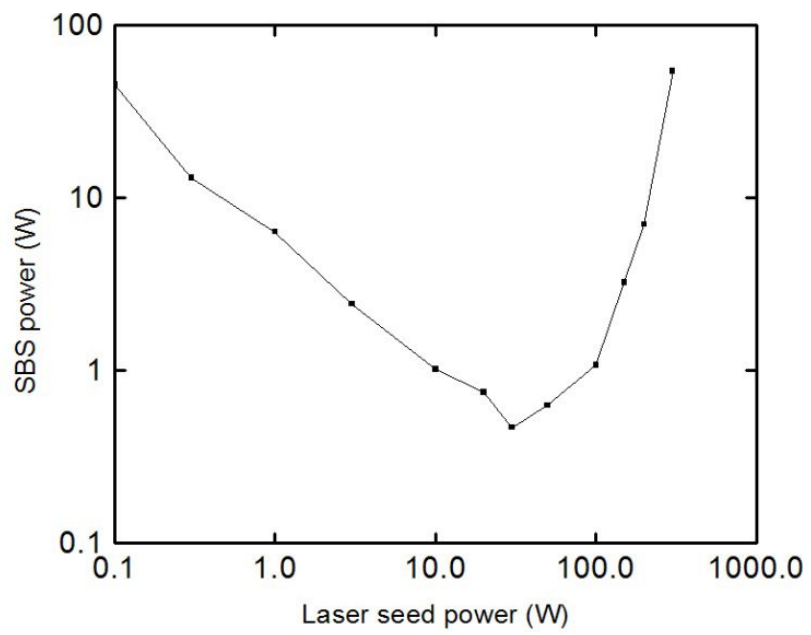

Fig. 8. SBS power vs. laser seed power averaged over 25 transit times. Minimum SBS occurs for a $30 \mathrm{~W}$ seed power.

\section{CONCLUSION}

A numerical model was developed that uses five coupled differential equations to describe SBS in a fiber amplifier seeded with a frequency-chirped laser. One advantage of using a linear frequency chirp is that the SBS threshold is insensitive to delivery fiber length. A robust numerical model can help in designing a fiber amplifier. This novel method of SBS suppression, along with our recent demonstration of coherent combining of multiple chirped seed amplifiers [8], demonstrates a path toward coherent combining of a large number of multi-kW fiber amplifiers to reach the $100 \mathrm{~kW}$ level. 


\section{REFERENCES}

[1] G. P. Agrawal, "Stimulated Brillouin Scattering", in Nonlinear Fiber Optics, San Diego: Academic Press, 1995.

[2] C. Zeringue, I. Dajani, S. Naderi, G. T. Moore, C. Robin, "A theoretical study of transient stimulated Brillouin scattering in optical fibers seeded with phase-modulated light," Opt. Express vol. 20, no. 19, pp. 21196-21213, (2012).

[3] R.W. Boyd, K. Rzazewski, and P. Narum, "Noise initiation of stimulated Brillouin scattering," Phys. Rev. A vol. 42 , no. 9, 5514-5521, 1990.

[4] E. Petersen, Z. Y. Yang, N. Satyan, A. Vasilyev, G. Rakuljic, A. Yariv, J. O. White, "Stimulated Brillouin Scattering Suppression With a Chirped Laser Seed: Comparison of Dynamical Model to Experimental Data," IEEE J. Quantum Electron. vol. 49, no. 12, pp. 1040-1044 2013.

[5] J. O. White, A. Vasilyev, J.P. Cahill, N. Satyan, O. Okusaga, G. Rakuljic, C.E. Mungan, A. Yariv, "Suppression of stimulated Brillouin scattering in optical fibers using a linearly chirped diode laser," Opt. Express vol. 20, no. 14, pp. 15872-15881, 2012.

[6] N. Satyan, A. Vasilyev, G. Rakuljic, J. O. White, A. Yariv, "Phase-locking and coherent power combining of broadband linearly chirped optical waves," Optics Express, vol. 20, no. 23, pp. 25213-25227, 2012.

[7] G. D. Goodno, S. J. McNaught, J. E. Rothenberg, T. S. McComb, P. A. Thielen, M. G. Wickham, M. E. Weber, "Active phase and polarization locking of a $1.4 \mathrm{~kW}$ fiber amplifier," Opt. Lett. vol. 35, no. 10, pp. 1542-1544, 2010.

[8] A. Vasilyev, E. Petersen, N. Satyan, G. Rakuljic, A. Yariv, "Coherent power combining of chirped-seed erbiumdoped fiber amplifiers," IEEE Photon. Technol. Lett. Vol. 25, no. 16, pp. 1616-1618, 2013. 